\title{
Prognosis for metastatic colorectal cancer patients achieving complete response after systemic chemotherapy
}

Takahiro Manabe ( $\nabla$ straight_no_chaser_tm@yahoo.co.jp)

Niigata cancer center hospital https://orcid.org/0000-0002-7199-6883

\section{Yasumasa Takii}

Niigata cancer center hospital

Hidehito Oyanagi

Niigata cancer center hospital

Hitoshi Nogami

Niigata cancer center hospital

\section{Satoshi Maruyama}

Niigata cancer center hospital

\section{Research Article}

Keywords: $\mathrm{mCRC}, \mathrm{CR}$, prognosis, unresectable lesions

Posted Date: February 22nd, 2022

DOI: https://doi.org/10.21203/rs.3.rs-651979/v2

License: (c) (i) This work is licensed under a Creative Commons Attribution 4.0 International License. Read Full License 


\section{Abstract}

Purpose: Despite marked recent advances in chemotherapy, few reports have focused on the prognosis for patients with metastatic colorectal cancer (mCRC) achieving complete response (CR) after systemic chemotherapy. This study investigated the clinical course of $\mathrm{MCRC}$ patients achieving $\mathrm{CR}$ and evaluated the role of $\mathrm{CR}$ in chemotherapy.

Methods: This retrospective study searched a prospectively maintained database at the author's institute to identify medical records for $\mathrm{MCRC}$ patients achieving CR after systematic chemotherapy from January 2007 to March 2020.

Results: The search yielded 23 patients with confirmed CR to systemic chemotherapy. Median time to CR from treatment initiation was 6.8 months. Maintenance chemotherapy was continued for 22 of 23 patients. Median duration of maintenance chemotherapy was 11.1 months. Disease progression occurred for 17 (73.9\%) patients at a median 48.1-month follow-up. Median progression-free survival was 26.6 months. Median overall survival was 91.7 months.

Conclusions: Patients with CR to chemotherapy had a high probability of disease progression, but a relatively long-term prognosis. Treatment strategies after achievement of CR should be based an understanding of the high potential that tumor cells will remain. Use of maintenance chemotherapy after achievement of $\mathrm{CR}$ is still unclear, the recent data do not demonstrate a negative impact for continuing maintenance chemotherapy after $\mathrm{CR}$.

\section{Introduction}

In Japan, $>137,000$ cases of colorectal cancer (CRC) are diagnosed annually; approximately $20 \%$ of these patients will have synchronous distant metastasis.[1, 2] An estimated $75-90 \%$ of patients with distant metastasis have unresectable lesions.[3] With best supportive care, median survival time (MST) for patients with unresectable CRC is approximately 8 months,[4] and advances in systemic chemotherapy have improved MST to approximately 30 months. $[5,6]$ Complete response (CR) rate is also improving, although still low. In the 1990s, the backbone of systematic chemotherapy in metastatic colorectal cancer (mCRC) was 5 -fluorouracil with leucovorin, which yielded only a $<1 \%$ CR rate. In the 2000 s, standard systemic chemotherapy for $\mathrm{MCRC}$ was 5 -fluorouracil-based chemotherapy in combination with new cytotoxic drugs, oxaliplatin or irinotecan, which improved the $\mathrm{CR}$ rate to $4-6 \%$. Furthermore, a higher $\mathrm{CR}$ rate of $8 \%$ has been reported for triplet chemotherapy FOLFOXIRI.[7-10] Despite these improvements, few reports have addressed the clinical course of patients achieving CR. This study evaluated the clinical course of $\mathrm{mCRC}$ patients achieving $\mathrm{CR}$ after systematic chemotherapy and the role of $\mathrm{CR}$ in chemotherapy.

\section{Methods}


This retrospective study searched a prospectively maintained database at the author's institute to identify medical records for $\mathrm{mCRC}$ patients achieving CR after systematic chemotherapy from January 2007 to March 2020. For all patients, the histologic diagnosis was adenocarcinoma of the colon and rectum. The study enrolled only patients achieving CR with systematic chemotherapy, regardless of previous surgery. Exclusion criteria were surgery for curative attempt of residual metastases after systematic chemotherapy and achievement of CR after local chemotherapy (e.g., hepatic arterial infusion chemotherapy.) The study was conducted in accordance with the Declaration of Helsinki.

In the authors' hospital, treatment for mCRC was performed according to the Japanese Society for Cancer of the Colon and Rectum guidelines.[2, 11, 12] After initiation of systemic chemotherapy, tumor response was evaluated using computed tomography scan and magnetic resonance imaging scan every 2-3 months based on response evaluation criteria for solid tumors.

For patients who achieved $\mathrm{CR}$, the management plan was to continue maintenance chemotherapy with a goal of 6-12 months or until disease progression. Patients who continued maintenance chemotherapy without major adverse events and disease progression had the option of continuing of maintenance chemotherapy for $>12$ months. For patients with disease progression after achievement of $C R$, resectability of the metastatic lesions was evaluated. If resectable, then curative resection was performed. If unresectable, then systemic chemotherapy was selected.

\section{Statistical Analysis}

Statistical calculations were performed using EZR (Saitama Medical Center, Jichi Medical University, Saitama, Japan). Survival was calculated with the Kaplan-Meier method.

\section{Results}

\section{Patient Characteristics and Systematic Chemotherapy}

The search yielded 23 patients with confirmed CR to systemic chemotherapy. All patients had a primary tumor resection before starting systemic chemotherapy. Metastases in only 1 organ were noted for 15 patients (64.9\%): 7 were distant lymph nodes (30.3\%), 6 liver (26.0\%), 1 lung (4.3\%), and 1 peritoneum (4.3\%).

Metastases in 2 organs were noted for 8 patients (35.1\%), and no patients who had metastases lesions in $\geq 3$ organs had confirmed CR. Median time to CR from initiation of systemic chemotherapy was 6.8 months (3.0-31.6 months, Fig. 1). With first-line chemotherapy, 20 (87\%) patients had confirmed CR; with second-line or later chemotherapy, $3(13 \%)$ had confirmed CR. Table 1 lists characteristics of patients with CR to systematic chemotherapy. 
Table 1

Characteristics of patients with complete response to systematic chemotherapy

\begin{tabular}{|c|c|c|}
\hline & & All $(n=23)$ \\
\hline \multirow[t]{2}{*}{ Age } & Median & 61 \\
\hline & Range & $34-84$ \\
\hline \multirow[t]{2}{*}{ Sex } & M & $13(56.5 \%)$ \\
\hline & $\mathrm{F}$ & $10(43.5 \%)$ \\
\hline \multirow[t]{2}{*}{ Primary lesion site } & $\mathrm{R}$ & $10(43.5 \%)$ \\
\hline & L & $13(56.5 \%)$ \\
\hline \multirow[t]{2}{*}{ Disease status } & Initial diagnosis & $12(52.2 \%)$ \\
\hline & Recurrence & $11(47.8 \%)$ \\
\hline \multirow[t]{2}{*}{ Prior chemotherapy } & Yes & $2(8.7 \%)$ \\
\hline & No & $21(91.3 \%)$ \\
\hline \multirow[t]{9}{*}{ Metastatic lesions } & Liver only & $6(26.0 \%)$ \\
\hline & Lung only & $1(4.3 \%)$ \\
\hline & distant lymph nodes only & $7(30.4 \%)$ \\
\hline & peritoneum only & $1(4.3 \%)$ \\
\hline & Liver \& Lung & $2(8.7 \%)$ \\
\hline & Liver \& distant lymph nodes & $1(4.3 \%)$ \\
\hline & Liver \& peritoneum & $3(13.0 \%)$ \\
\hline & Lung \&distant lymph nodes & $2(8.7 \%)$ \\
\hline & distant lymph nodes \& peritoneum & $1(4.3 \%)$ \\
\hline \multirow[t]{3}{*}{ Line } & $1 \mathrm{st}$ & $20(87.0 \%)$ \\
\hline & 2nd & $2(8.6 \%)$ \\
\hline & $3 r d$ & $1(4.3 \%)$ \\
\hline \multirow[t]{4}{*}{ Regimen } & FOLFOX + BEV/PANI/CET & $9(39.1 \%)$ \\
\hline & Xelox + BEV & $8(34.8 \%)$ \\
\hline & FOLFIRI + BEV/PANI/CET & $1(4.3 \%)$ \\
\hline & IRIS + BEV & $2(8.7 \%)$ \\
\hline
\end{tabular}




\begin{tabular}{|ll|}
\hline & All $(\mathbf{n}=\mathbf{2 3})$ \\
\hline CPT11 + CET & $1(4.3 \%)$ \\
\hline FOLFOXIRI + BEV & $1(4.3 \%)$ \\
\hline 5 FU/LV & $1(4.3 \%)$ \\
\hline
\end{tabular}

Maintenance chemotherapy was continued for 22 of 23 patients; 1 patient had confirmed pathological CR. Disease progression occurred for 11 patients during maintenance chemotherapy, and 2 patients discontinued maintenance chemotherapy in $<6$ months due to adverse events. Median duration of maintenance chemotherapy was 11.1 months (1.7-36.8 months).

\section{Prognosis For Patients Achieving $\mathrm{Cr}$ To Systematic Chemotherapy}

Of the 23 patients with CR, $6(26.1 \%)$ maintained CR and 17 (73.9\%) had disease progression after a median follow-up of 48.1 months. Curative resection was achieved in 6 of the 17 patients with disease progression; of these 17 patients, 2 have remained disease free; 6 have continued treatment, and 9 had disease progression and died.

Median progression-free survival (PFS) was 26.6 months (95\% confidence interval [Cl] 17.7-29.6 months, Fig. 2). Median CR duration was 14.7 months ( $95 \% \mathrm{Cl} 8.7-22.9$ months). No disease progression was observed in patients who maintained CR for $>24.3$ months. Median overall survival (OS) was 91.7 months (95\% Cl 45.7-NA months; Fig. 3).

\section{Discussion}

Despite marked recent advances in chemotherapy, few reports have addressed the prognosis for mCRC patients achieving CR. Despite the small sample size, the results of this study are significant for understanding the clinical course of the patients achieving CR. Clinical outcomes were evaluated for 23 CRC patients confirmed CR to systematic chemotherapy. Results showed that patients with CR to chemotherapy had a high probability of disease progression, but a relatively long-term prognosis.

Although CR determined imaging is a useful criterion for evaluating the efficiency of chemotherapy, some liver metastasis studies have reported a discrepancy between CR visualized by imaging versus pathologically confirmed CR. Approximately $20-25 \%$ of liver metastases disappear after systemic chemotherapy; among such disappearing liver metastasis (DLM), "true CR" has been reported in 33-83\% of cases. Therefore, resection is recommended for DLM after chemotherapy.[13-16] In the present study, 17 (73.9\%) patients with CR to systematic chemotherapy had disease progression after a median followup duration of 48.1 months. Another study also reported that $84 \%$ of patients with CR had disease 
progression.[8] These data indicate a discrepancy between CR determined by imaging and actual complete clearance of cancer in other organs.

Use of maintenance chemotherapy after achievement of CR is unclear. Research has shown negative results for maintenance chemotherapy after achievement of CR. Dy GK et al compared OS in 22 of 62 patients with CR with $<2$ cycles of maintenance chemotherapy and 40 patients with $>2$ cycles of chemotherapy in 2007. As a result, OS from CR was 39.8 months and 29.2 months, respectively; maintenance chemotherapy did not prolong OS.[8] However, the therapeutic background for the 2007 study differs from the present context. Treatment strategies after achievement of CR have not been established; the approach should be based on the high potential for tumor cells to remain even after CR. Furthermore, the OPTIMOX2 study, which compared chemotherapy discontinuation with maintenance therapy, indicated that planned complete discontinuation of chemotherapy had a negative impact on duration of disease control and PFS versus the maintenance therapy strategy.[17] Therefore, in the present study, maintenance chemotherapy was continued until disease progression or for 6-12 months after $\mathrm{CR}$, except for cases in which pathological CR was confirmed after surgery for DLM. Median duration of maintenance chemotherapy was 11.1 months.

In the present study, median PFS was 26.6 months and median OS was 91.7 months, which exceeds the PFS (15.4 months) and OS (44.3 months) in the 2007 study.[8] These more recent findings indicate that advances in chemotherapy have improved the prognosis of patients with $\mathrm{CR}$; however, direct comparison with the 2007 study is difficult results because of different therapeutic backgrounds, and the recent data do not demonstrate a negative impact for continuing maintenance chemotherapy after CR.

This study has some limitations. First, a relatively small patient sample was derived from a single institution. Second, systematic chemotherapy regimens were not uniformly introduced, which result in treatment bias.

\section{Conclusions}

Although CR by imaging is a useful criterion for evaluating efficiency of chemotherapy, it does not mean actual complete clearance of cancer. Treatment strategies after the achievement of CR should be decided based on the understanding of a high possibility that tumor cells will remain even if the patient has achieved CR.

\section{Abbreviations}

$\mathrm{CR}$, complete response; CRC, colorectal cancer; DLM, disappearing liver metastasis; JSCCR, Japanese Society for Cancer of the Colon and Rectum; MCIJ, monitoring of cancer incidence in Japan; MST, median survival time; OS, overall survival; PFS, progression-free survival 


\section{Declarations}

\section{Acknowledgements}

The authors would like to thank Enago (www.enago.jp) for the English language review.

\section{Funding}

The authors did not receive any funding.

\section{Competing interests}

The authors declare that they have no conflicts of interest.

\section{Authors' contributions}

Takahiro Manabe wrote the initial draft of the manuscript. Yasumasa Takii was involved in the preparation of the manuscript. All other authors critically reviewed the manuscript. All authors approved the final version of the manuscript and agree to be accountable for all aspects of the work in ensuring that questions related to the accuracy or integrity of any part of the work are appropriately investigated and resolved.

\section{Availability of data and materials}

The datasets analyzed during the current study are available from the corresponding author on reasonable request.

\section{Ethics approval}

The study procedures were conducted in accordance with the Declaration of Helsinki. Written informed consent was obtained from the patient for publication of this studies before study inclusion.

\section{References}

1. Hori M, Matsuda T, Shibata A, Katanoda K, Sobue T, Nishimoto H, et al. Cancer incidence and incidence rates in Japan in 2009: a study of 32 population-based cancer registries for the Monitoring of Cancer Incidence in Japan (MCIJ) project. Jpn J Clin Oncol. 2015;45:884-91.

2. Watanabe T, Itabashi M, Shimada Y, Tanaka S, Ito Y, Ajioka Y, et al. Japanese society for cancer of the colon and rectum (JSCCR) guidelines 2014 for treatment of colorectal cancer. Int J Clin Oncol. 2015;20:207-39.

3. Martin R, Paty P, Fong Y, Grace A, Cohen A, DeMatteo R, et al. Simultaneous liver and colorectal resections are safe for synchronous colorectal liver metastasis. J Am Coll Surg. 2003;197:233-42.

4. Simmonds PC. Palliative chemotherapy for advanced colorectal cancer: systematic review and metaanalysis. Colorectal Cancer Collaborative Group BMJ. 2000;321:531-35. 
5. Yamada Y, Takahari D, Matsumoto H, Baba H, Nakamura M, Yoshida K, et al. Leucovorin, fluorouracil, and oxaliplatin plus bevacizumab versus S-1 and oxaliplatin plus bevacizumab in patients with metastatic colorectal cancer (SOFT): an open-label, non-inferiority, randomised phase 3 trial. Lancet Oncol. 2013;14:1278-186.

6. Yamazaki K, Nagase M, Tamagawa H, Ueda S, Tamura T, Murata K, et al. Randomized phase III study of bevacizumab plus FOLFIRI and bevacizumab plus mFOLFOX6 as first-line treatment for patients with metastatic colorectal cancer (WJOG4407G). Ann Oncol. 2016;27:1539-46.

7. Rothenberg ML, Oza AM, Bigelow RH, Berlin JD, Marshall JL, Ramanathan RK, et al. Superiority of oxaliplatin and fluorouracil-leucovorin compared with either therapy alone in patients with progressive colorectal cancer after irinotecan and fluorouracil-leucovorin: interim analysis of a phase III trial. J Clin Oncol. 2003;21:2059-69.

8. Dy GK, Krook JE, Green EM, Sargent DJ, Delaunoit T, Morton RF, et al. Intergroup N9741: impact of complete response to chemotherapy on overall survival in advanced colorectal cancer: results from Intergroup N9741. J Clin Oncol. 2007;25:3469-74.

9. Bokemeyer C, Bondarenko I, Makhson A, Hartmann JT, Aparicio J, de Braud F, et al. Fluorouracil, leucovorin, and oxaliplatin with and without cetuximab in the first-line treatment of metastatic colorectal cancer. J Clin Oncol. 2009;27:663-71.

10. Falcone A, Ricci S, Brunetti I, Pfanner E, Allegrini G, Barbara C, et al. Phase III trial of infusional fluorouracil, leucovorin, oxaliplatin, and irinotecan (FOLFOXIRI) compared with infusional fluorouracil, leucovorin, and irinotecan (FOLFIRI) as first-line treatment for metastatic colorectal cancer: the Gruppo Oncologico Nord Ovest. J Clin Oncol. 2007;25:1670-76.

11. Watanabe T, Muro K, Ajioka Y, Hashiguchi Y, Ito Y, Saito Y, et al. Japanese Society for Cancer of the Colon and Rectum (JSCCR) guidelines 2016 for the treatment of colorectal cancer. Int J Clin Oncol. 2018;23:1-34.

12. Hashiguchi Y, Muro K, Saito Y, Ito Y, Ajioka Y, Hamaguchi T, et al. Japanese Society for Cancer of the Colon and Rectum (JSCCR) guidelines 2019 for the treatment of colorectal cancer. Int J Clin Oncol. 2020;25:1-42.

13. van Vledder MG, de Jong MC, Pawlik TM, Schulick RD, Diaz LA, Choti MA. Disappearing colorectal liver metastases after chemotherapy: should we be concerned? J Gastrointest Surg. 2010;14:1691700 .

14. Auer RC, White RR, Kemeny NE, Schwartz LH, Shia J, Blumgart LH, et al. Predictors of a true complete response among disappearing liver metastases from colorectal cancer after chemotherapy. Cancer. 2010;116:1502-09.

15. Benoist S, Brouquet A, Penna C, Julie C, EL Hajjam M, Chagnon S, et al. Complete response of colorectal liver metastases after chemotherapy: does it mean cure? J Clin Oncol. 2006;24:3939-45.

16. Allen PJ, Kemeny N, Jarnagin W, DeMatteo R, Blumgart L, Fong Y. Importance of response to neoadjuvant chemotherapy in patients undergoing resection of synchronous colorectal liver metastases. J Gastrointest Surg. 2003;7:109-17. 
17. Chibaudel B, Maindrault-Goebel F, Lledo G, Mineur L, Andre T, Bennamoun M, et al. Can chemotherapy be discontinued in unresectable metastatic colorectal cancer? The GERCOR OPTIMOX2 Study. J Clin Oncol. 2009;27:5727-33.

\section{Figures}

Figure 1

Time to complete response from initiation of systemic chemotherapy

Figure 2

Progression-free survival

Figure 3

Overall survival 